\title{
Authentification of Einstein's Static Universe of 1917
}

\author{
Eli Peter Manor \\ Israel Medical Association, Caesarea, Israel \\ Email: dr.eli.manor@gmail.com
}

Received 2 October 2014; revised 1 November 2014; accepted 28 November 2014

Copyright (C) 2014 by author and Scientific Research Publishing Inc.

This work is licensed under the Creative Commons Attribution International License (CC BY). http://creativecommons.org/licenses/by/4.0/

\section{(c) (i) Open Access}

\begin{abstract}
Static cosmology has been abandoned almost a century ago because of phenomena which were unexplained at those times. However, that scenario can be revived with the modern findings of gravitational forces, coming from outside of the "luminous world", tugging on our universe. These unexplained phenomena were: the redshift, the CMB, and Olbers' paradox. All these can now be explained, as done in the present manuscript. 1) The observed redshift, which is commonly attributed to the Doppler effect, can also be explained as a gravitational redshift. Thus, the universe is not expanding, as has also been described in recent publications, thereby, making the "Big Bang" hypothesis unnecessary. 2) Gravitation is induced by matter, and at least some of the distant matter is expected to be luminous. That electro-magnetic emission is extremely redshifted, and thus perceived by us as CMB. CMB is not necessarily a historic remnant related to the "Big Bang", rather it is the redshifted light, coming from extremely distant luminous matter. 3) According to Olbers' paradox, the night sky is expected to be bright. The sky looks dark because the light coming from extremely faraway light sources, out of our visible universe, is extremely redshifted. Therefore it is perceived by us as CMB. As in the cosmological literature many problems with the "Big Bang" hypothesis have been described, where as the problems with the static universe model are resolved in the present manuscript, the static state scenario should be renovated.
\end{abstract}

\section{Keywords}

Big Bang Never Happened, CMB Is Redshifted Light, Gravitational Redshift, Dark Sky Illusion, Einstein, Static Universe

\section{Introduction}

Albert Einstein proposed a homogeneous isotropic static, temporally infinite model of the universe in 1917 [1], 
but then came the clamor for an expanding universe. Therefore he forwarded in 1931 a steady-state dynamic universe in expansion [2] [3]. This expanding model requires a mechanism to recreate matter and it has also been abandoned [4]. To Einstein, a static universe required a force equal, but opposite to the gravity, produced by the matter of the universe to avoid total collapse. He named this repelling force the "cosmological constant".

Years thereafter, the static and expanding steady state cosmological models were considered obsolete. The prevailing cosmological model became the Big Bang hypothesis. However, there are many flaws with this scenario [5] [6]. Therefore, other models must also be considered.

Since those times, at the beginning of the $20^{\text {th }}$ century, many new cosmological findings have been observed. Among those are the description of the dark matter and the pulling of extra-universal forces on our universe, including the description of parallel universes. Parallel worlds, termed multiverse, or in other words—an infinite, endless universe with indefinite matter-will exert gravity all around us. This endless, infinite gravitational force pulls on our universe in all directions. The universe is tugged by this gravity coming from outside the observable universe [7]-[9]. It is indicative that observed tilt exerted across the entire horizon of our universe comes from a faraway source [10]. Problems exist with the Big Bang hypothesis, however, new cosmological findings were discovered since Einstein's days, hence, the static world must be reconsidered.

In order to verify Einstein's static cosmological model of 1917, the three following observations must be explained:

1) It must explain the intergalactic redshift.

2) It must explain the source of the cosmic microwave background.

3) It must explain why the night sky appears black between stars and galaxies.

Herewith are explanations to all these three cosmological observations by which the static universe model can be renovated.

\section{Explaining the Intergalactic Redshift}

The first observation that must be explained is the redshift theme. The mainstream explanation is the Doppler shift, and therefore, the hypothesis of an expanding universe. However, many other cosmological explanations have been described. Even Hubble, who discovered this redshift, warned about jumping to conclusions that this universe was actually expanding.

One of the oldest and prominent alternative theories is the gravitational inspired redshift. The influence of gravity on light has been proposed by Albert Einstein already in 1911 [11]. Another interpretation of gravitational redshift is by quantum celestial mechanics in which no space expansion is necessary [12]. Gravitational redshift has been described as the electromagnetic influence from gravity on photons. Energy is transferred from the light wave when it propagates from the gravitational body ensuing in the light's redshift [13].

As light moves out of a gravitational field, it must lose energy because it works against that gravitational field. As photons always travel at the speed of light, the only way this energy loss can show up is in the increase of wavelength (or decrease in frequency). Distant objects, like those tugging at our universe [7]-[10], have a gravitational effect, as has dark matter. These tremendous gravitational forces can cause light to be redshifted.

Although it is generally accepted that the mass of dark matter is over $95 \%$ of the mass of the universe, its gravitational redshift effect on light is considered to be too weak to account for the observed redshift. However, if the gravitational force coming from outside the "luminous matter" is infinite and immense, it becomes evident that it is able to produce the observed redshift. The notion that gravitational forces that tug on our universe exist outside the observable universe, is already well accepted in the cosmological community [7]-[10].

If one includes the matter of an infinite universe (multiverse), the "luminous matter" comes to be only a fraction of the total mass, so the visible universe is less than the usually described $4.9 \%$ of all matter.

The redshift can be approximated by the binominal expansion to become:

$$
Z_{\text {approx }}=\frac{G M}{c^{2} R}
$$

where $Z$ is the redshift, $G$ is Newton's gravitational constant, $M$ is the mass exerting the gravity and $R$ is the distance between the peak of the gravitational effect to the photons source. When $M$ becomes almost indefinite ( $\infty$ ), it obviously is able to redshift any light. Therefore it can account for the observed redshift.

The galaxies which are farther away from the Milky Way are more affected by the redshift caused by the parallel worlds, because their distance $(R)$ to those out-of-the-visible-world forces, is shorter. The galaxies nearer 
to the Milky Way will appear less redshifted, as their distance $(R)$ from the main gravitational field is larger, so their redshifting effect diminishes.

In an isotropic, infinite, endless cosmos the gravitational forces coming from all directions are expected to be similar. It turns out that the gravitational effect of our own vicinity is extremely minor and negligible in comparison to that of the endless multiverse. The gravitational influence coming from any direction is countered by the pull coming from the opposite direction. The redshift effect vanishes altogether due to the pull coming from the opposite direction, and that location is close to our universe.

The evidence that the average gravitational force coming from the extra-celestial gravitation is stronger than that of our universe is the observation that it pulls on clusters within our universe [10]. In other words, the magnitude of the redshift decreases as the distance $(R)$ of the photons from the peak of the gravitational force which tugs on our universe, increases. That is, that its distance from us decreases. This explains Hubble's finding that the redshift increases in proportion to the increase of distance of the light source from us.

Photons approaching the Milky Way might balance, or even outweigh this redshift effect. Therefore they are even blueshifted, as is the case with the Virgo cluster including the Andromeda galaxy. Photons departing from a gravitational field are redshifted whereas those approaching such fields are blueshifted. These blueshifted galaxies cast doubts on the expanding universe theory. Therefore gravitational red and blue shifts must be reconsidered [14]. The observed redshifts give the illusion that an object is receding away, when in fact an extremely immense mass is causing that redshift.

Summing up the redshift issue, there exist several alternatives to the Doppler shift. Another alternative is the gravitational redshift from a tremendously immense gravitational force induced by endless matter, much greater than the observable matter.

\section{Explaining the Source of CMB}

The second observation that must be explained is the CMB topic. The prevailing cosmological explanation of the CMB is that it is a historic remnant of the "light emitted by the Big Bang" and is assumed to be its thermal residue. CMB is an electromagnetic radiation with its brightest wavelength of about $1.9 \mathrm{~mm}$. Therefore it is possibly an extremely redshifted light, coming from parallel worlds.

Considering that a multitude of innumerable, endless universes exist all around our universe, and their electromagnetic emission includes light like that emitted by our universe, and that light is extremely redshifted, it will be perceived by us as CMB. This very extreme redshift may be the result of the departure of the photons from the tremendously strong gravitational force caused by the countless, infinite parallel worlds all around our universe.

The light emitted by parallel worlds will have a wavelength like that of any star. As said, that light will be extremely redshifted by gravity of those parallel worlds, so it will reach us in the CMB wavelength. To calculate this redshift the sun was chosen arbitrarily, but any star could be chosen.

The peak wavelength emitted by the sun is considered to be $\lambda$ source $=483 \mathrm{~nm}$. The peak wavelength of the CMB is about $\lambda_{\text {obs }}=1.9 \mathrm{~mm}$. So the redshift, which is commonly denoted by " $Z$ " is:

$$
Z=\frac{\lambda_{\text {obs }}-\lambda_{\text {source }}}{\lambda_{\text {source }}}=3932.7474 \text {. }
$$

CMB is a key prediction of the hot Big Bang theory and an important observation that discriminates between the "Big Bang" and the static world scenario. The recently detected ripples in the CMB, attributed to gravitational waves, also lend credence to the idea of a multiverse [15]. Gravitational waves coming from outside the visible universe can easily produce ripples. Therefore the conception of a static, endless, infinite universe must be revived.

\section{Explaining the Dark Night Sky}

The third observation that must be explained is the "dark night sky paradox". The sky appears black between the stars and galaxies. It is said that if the universe is static, homogeneous at a large scale, and populated by an infinite number of stars, any sightline from earth must end at the surface of a star. So the sky at night should appear completely bright, not black. This paradox was described by the astronomer Heinrich Wilhelm Olbers (17581840). This unexplained paradox is one of the evidences for a non-static universe and supports the hypothesis of 
an expanding universe. However, the dark sky is a completely false impression. The night sky is not dark at all, most of the light perceived by us is just out of the visual spectrum. The light emitted from very distant sources is extremely redshifted and reaches us as CMB, so we cannot see it. Therefore, we get the erroneous impression that the sky is dark. The extreme redshift of the light is the solution of Olbers' paradox.

\section{Conclusions}

The Big Bang theory, in spite of being the mainstream hypothesis, is very problematic [5] [6]. Hence, other models should also be considered. These include the old Einstein's 1917 static world scenario. In order to revive this static state model of the universe, its old problems must be resolved. This is done in this manuscript and has the consequent implications:

- The redshift has been explained by gravitational forces which tug on our universe [10]. It has already been reported that observational evidence favors a static universe and that the universe is not expanding [16] [17].

- The CMB is redshifted light emitted by sources out of the observable universe, and Olber's paradox is just an illusion.

- The light emitted from distant light sources is extremely redshifted. Therefore it is perceived on earth as CMB which is out of our visible wavelength.

In conclusion, alternative explanations for the redshift, CMB and Olbers' paradox, which are the cornerstones of the "Big Bang" hypothesis, are presented in this paper. By solving the problems with the static state model of the universe, this model can be renovated. Therefore the "Big Bang" may never have happened.

The static state model does not require the creation of a singularity from nothing, and does not have the many problems described with the Big Bang hypothesis. So the static world model again becomes a credible belief as it was in 1917. Although much evidence accumulated against the Big Bang hypothesis, it is still widely accepted. Therefore, the significance of the present paper is in solving the problems with the alternative model, the static scenario.

\section{References}

[1] Einstein, A. (1917) Sitzung der Physikalisch-Mathematischen Klasse, 8, 142-152.

[2] Einstein, A. (1931) Zum Kosmologischen Problem. Archive Online, Archive No. 2-112. http://alberteinstein.info/vufind1/Record/EAR000034354

[3] Einstein, A. (1931) Zum kosmologischen Problem der allgemeinen Relativitätstheorie. Sitzungsb. A Kad, König Preuss, 235-237.

[4] Einstein's Steady-State Theory: An Abandoned Model of the Cosmos. Cormac O'Raifeartaigh, Brendan McLann, Werner Nahm and Simon Mitton.

[5] van Flandern, T. (2002) Meta Research Bulletin, 11, 6-13.

[6] Merali, Z. (2013) Higgs Data Could Spell Trouble for Leading Big Bang Theory. Nature/News I.

[7] Roach, J. (2010) New Proof of Unknown “Structure” Tug at Our Universe. National Geographic, Daily News.

[8] Catchpole, H. (2009) Weird Data, Suggests Something Big beyond the Edge of the Universe. Cosmos Magazine.

[9] (2013) Other Universes Are Pulling on Our Universe. The Daily Galaxy, Great Discoveries Channel.

[10] Kashlinsky, A., Barandela, F.A., Kocevski, F. and Ebeling, H. (2009) Astrophysical Journal Letters, 686, 149-152.

[11] Einstein, A. (1911) Annalen der Physik 35, 898-908. http://dx.doi.org/10.1002/andp.19113401005

[12] Potter, F. and Preston, H.G. (2007) Progress in Physics, 2, 31-33.

[13] Giertz, H.W. (2014) International Journal of Astronomy and Astrophysics, 4, Article ID: 46666.

[14] http://www.poyntsource.com/Richard/blueshifted galaxies.htm

[15] Mersini-Houghton, L. (2014) Birth of the Universe from the Multiverse, MG13. arXiv: 1406.6275.

[16] Crawford, D.F. (2011) The Journal of Cosmology, 13.

[17] Lerner, E.J., Faloma, R. and Scarpa, R. (2014) International Journal of Modern Physics D, 23. 
Scientific Research Publishing (SCIRP) is one of the largest Open Access journal publishers. It is currently publishing more than 200 open access, online, peer-reviewed journals covering a wide range of academic disciplines. SCIRP serves the worldwide academic communities and contributes to the progress and application of science with its publication.

Other selected journals from SCIRP are listed as below. Submit your manuscript to us via either submit@scirp.org or Online Submission Portal.
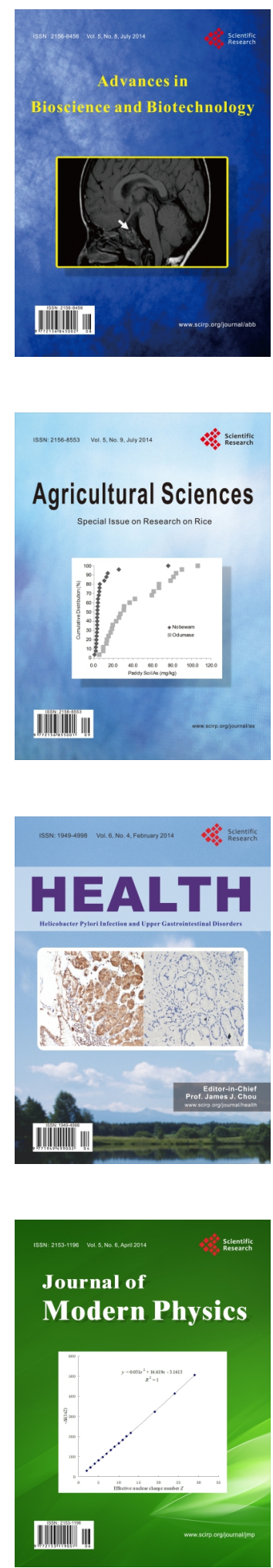
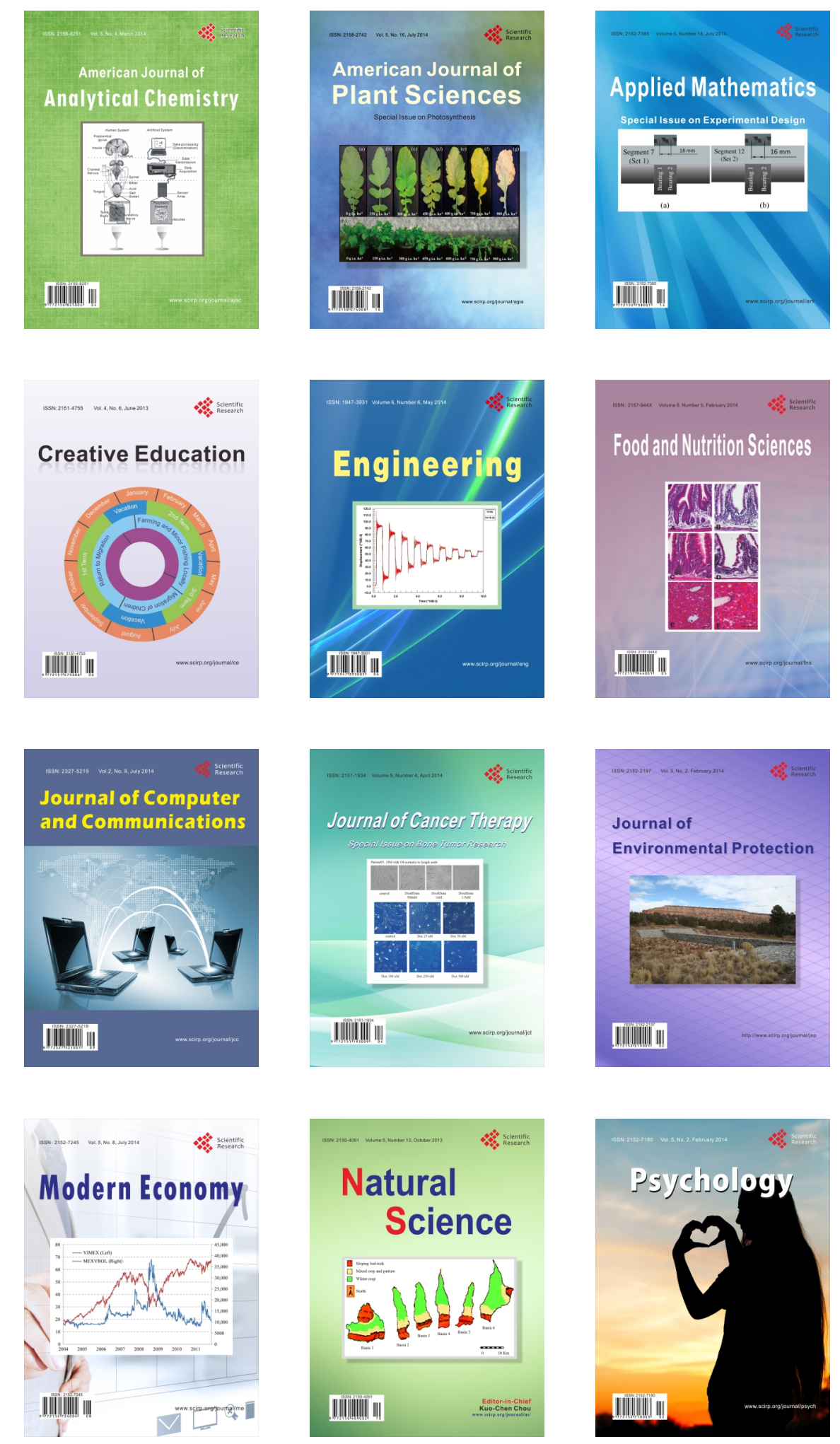